\title{
Estimation of depth to top of magnetic sources using the local-wavenumber approach in an area of shallow Moho and Curie depth - The Red Sea
}

\author{
Ahmed Salem¹, Richard Blakely², Chris Green ${ }^{1}$, Derek Fairhead ${ }^{1}$, and Dhananjay Ravat ${ }^{3}$
}

\begin{abstract}
The local-wavenumber method estimates the depth to a magnetic source based on the spectral content of a single anomaly assuming that the base of the magnetic body is at infinite depth. However, the "infinite-depth" assumption can lead to significant underestimation of the depth to the top of magnetic bodies, especially in areas where the depth to the bottom of the magnetic layer is not large compared to the depth to the top, as would occur in high heat-flow regions and thinned continental margins. Such underestimation of depths has been demonstrated in model studies and using real data with seismic and well control. We evaluated a modification to the local-wavenumber approach to estimate the depth to the top of magnetic sources assuming that the depth to the bottom of the magnetic sources is controlled by the Curie temperature or crustal thickness. We applied this new method to a simple model of a continental margin and to magnetic survey data over the central Red Sea where the Curie isotherm is shallow. The effective structural index of this finite depth extent model is found to increase continuously from the continent to the ocean as the depth to the magnetic basement increases and the depth to the bottom of the magnetic layer decreases. We have also discovered in this study that the local-wavenumber maxima correlate well with major seafloor spreading magnetic reversal epochs in the central Red Sea segment.
\end{abstract}

\section{Introduction}

Many methods of estimating the depth to basement from magnetic data rely on the simple assumption that the geometry of the causative source has an infinite depth extent (Flanagan and Bain, 2013). This assumption ignores the fact that the thickness of the magnetized layer will be depth limited by either the crust-mantle interface (the Moho) or the Curie temperature of the magnetic minerals, whichever depth is shallower (Wasilewski et al., 1979; Wasilewski and Mayhew, 1992; Lee et al., 2010; Salem et al., 2010; Flanagan and Bain, 2013). Within continental areas, the Moho and $\mathrm{Cu}-$ rie isotherm are likely to be at great depth and hence the effect on depth-to-source estimates is small. However, in stretched continental margins, the Curie temperature (the temperature at which rocks become nonmagnetic - generally considered to be around $580^{\circ} \mathrm{C}$ ) is relatively shallow and the depth to the top of magnetic sources can be significantly underestimated. Such underestimation can have significant interpretation consequences, for example, on petroleum prospectivity; this is a particular problem in areas where the basement is poorly resolved by seismic data
- e.g., beneath salt. Due to the size of the exploration data sets, automated grid-based methods tend to be favored for efficiency reasons. One popular automated method is Euler deconvolution (Reid et al., 1990), which produces depth estimates derived from overlapping windows generating multiple solutions of varying quality that need further processing to define a robust subset of solutions for interpretation. Following Salem et al. (2010), we focus on automatic grid-based methods that select individual anomalies to derive depth estimates, such as the tilt-depth method (Salem et al., 2007) or the local-wavenumber or source parameter imaging method after Thurston and Smith (1997). The theory behind these methods uses source models with the bottom at an infinite depth. The problems of ignoring the depth to bottom are highlighted by Lee et al. (2010) and Salem et al. (2010). This is further addressed by Flanagan and Bain (2013) using sets of nomographs to correct a range of classic and more recent single anomaly source methods to incorporate the finite depth to bottom.

Our approach in this paper is different in that it is based on adapting the theory to a model with finite

\footnotetext{
${ }^{1}$ Getech, Leeds, UK and University of Leeds, Leeds, UK. E-mail: ahmedsalem30@yahoo.com; cmg@getech.com; jdf@getech.com.

${ }^{2}$ United States Geological Survey, Menlo Park, California, USA. E-mail: blakely@usgs.gov.

${ }^{3}$ University of Kentucky, Lexington, Kentucky, USA. E-mail: dhananjay.ravat@uky.edu.

Manuscript received by the Editor 23 December 2013; revised manuscript received 6 February 2014; published online 5 June 2014. This paper appears in Interpretation, Vol. 2, No. 4 (November 2014); p. SJ1-SJ8, 8 FIGS.

http://dx.doi.org/10.1190/INT-2013-0196.1. @ 2014 Society of Exploration Geophysicists and American Association of Petroleum Geologists. All rights reserved.
} 
depth extent. This then removes the need to apply an extra stage of depth corrections to the depth estimate based on the infinite depth model. This approach has already been used to demonstrate a finite depth extent version of the tilt-depth method (Salem et al., 2013), whereas the present contribution presents a modification to the local-wavenumber method to deal with layers with a finite depth extent.

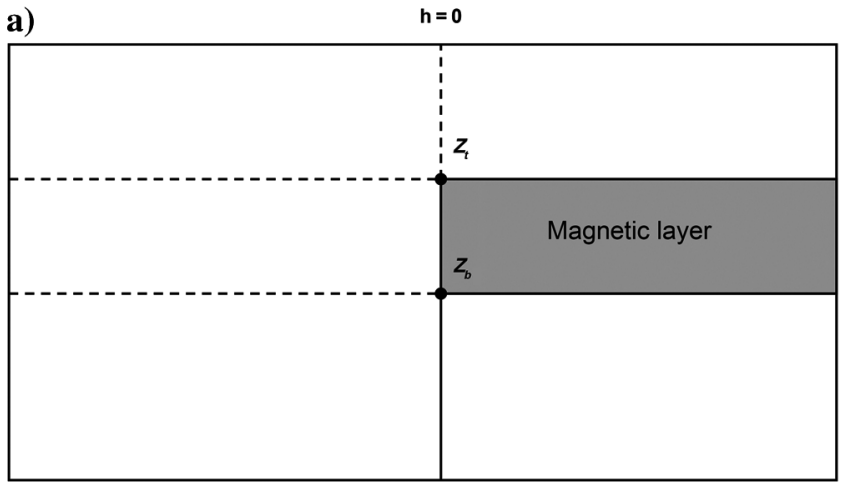

b)
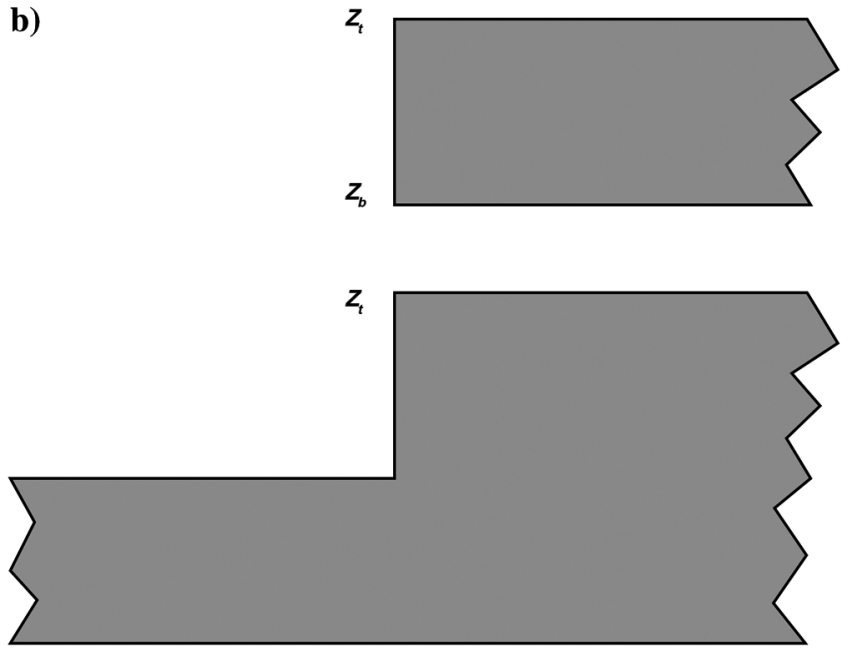

c)
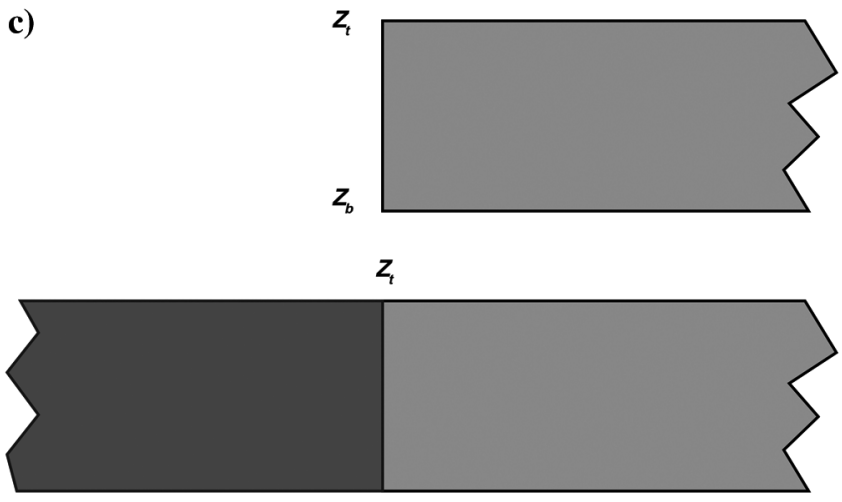

$z_{b}$

Figure 1. Conceptual geologic model represented by (a) a magnetic layer with two corners located vertically in the same location. (b) Vertical fault with finite depth extent and (c) vertical contact between zones with different magnetization within a constant thickness magnetic layer.

\section{Theory}

The local-wavenumber approach requires secondorder derivatives of the total field and is used to estimate the depth to the top of buried magnetic bodies. The local wavenumber is defined as the horizontal gradient of the local phase (Thurston and Smith, 1997):

$$
l w=\frac{\partial}{\partial h} \tan ^{-1}\left(\frac{\partial M}{\partial z} / \frac{\partial M}{\partial h}\right)
$$

where $M$ is the magnetic field measured on a horizontal surface and $\frac{\partial M}{\partial h}$ and $\frac{\partial M}{\partial z}$ are the horizontal and vertical derivatives of the field in the $h$ - and $z$-directions, respectively. The local wavenumber has a simple bell shape (similar to the shape of the total horizontal gradient), which peaks directly over certain simple 2D magnetic sources such as contacts, tops of thin dykes, and horizontal cylinders. For 2D magnetic sources with infinite depth extent, the local wavenumber at the peak is given by

$$
l w_{(h=0)}=\frac{\eta+1}{z_{t}}
$$

where $z_{t}$ is the depth to the top of the body and $\eta$ is a value characterizing source geometry (Salem and Smith, 2005). The parameter $\eta$ is equivalent to the structural index of the Euler method (Thompson, 1982); $\eta=$ 0 for a contact and 1 for a dike. In the conventional local-wavenumber approach, we assume the structural index and estimate the depth directly using equation 2 . In this paper, we investigate the use of the local-wavenumber approach in interpreting magnetic data over magnetic bodies having a range of finite depth extents. We use a finite magnetic layer with depth to top $z_{t}$ and depth to bottom $z_{b}$ (Figure 1a) - the "finite-contact" model. Following Nabighian (1972) and Blakely (1995), the equations for the horizontal and vertical derivatives of the total magnetic field $M$ over a finite vertical magnetic contact are given by

$$
\frac{\partial M}{\partial h}=K\left(\frac{z_{t} \cos \theta+h \sin \theta}{h^{2}+z_{t}^{2}}-\frac{z_{b} \cos \theta+h \sin \theta}{h^{2}+z_{b}^{2}}\right)
$$

and

$$
\frac{\partial M}{\partial z}=K\left(\frac{h \cos \theta-z_{t} \sin \theta}{h^{2}+z_{t}^{2}}-\frac{h \cos \theta-z_{b} \sin \theta}{h^{2}+z_{b}^{2}}\right),
$$

where $h$ is the horizontal distance from the top of the contact, $K$ is a constant related to the magnetic field and susceptibility of the magnetic layer, and $\theta=2 I-\pi$ is an angle derived from the magnetic inclination $i$ and the horizontal angle between the profile and magnetic north $A$ where $I=\tan i / \cos A$. Substituting equations 3 and 4 in equation 1 , the value of the local wavenumber directly above a vertical contact $(h=0)$ is expressed as 


$$
l w_{(h=0)}=\frac{1}{z_{t}}+\frac{1}{z_{b}} .
$$

Equation 5 represents a modification to the conventional local-wavenumber approach (equation 2). For a finite contact, the value of the local wavenumber at $h=$ 0 will be larger than for an infinite contact with the same $z_{t}$, such that if we apply equation 2 , which represents an infinite contact $(\eta=0)$, the depth to top will be underestimated.

The finite thickness contact model of Figure 1a can easily be used to represent two different simple conceptual geologic models. Figure $1 \mathrm{~b}$ shows how the semiinfinite slab is equivalent to a vertical basement fault (nonmagnetic layer above, magnetic layer below) of finite offset. This could be used to represent major basinbounding faults (where $z_{t}$ is the depth to the top of the fault and $z_{b}$ is the depth to the base of the fault), which might otherwise be interpreted based on models with $\eta=0$. The simple conceptual model considered in this paper is a vertical contact between two crustal zones (Figure 1c). In this model, the depths to the top $z_{t}$ and bottom $z_{b}$ of the layer are constant, but the magnetization is different on either side of the contact - and in fact, one of the sides could be nonmagnetic.

\section{Relationship between the depth extent and the structural index}

To understand the relation between model thickness and structural index $\eta$, we express the basal depth as a function of the top depth; i.e.,

$$
z_{b}=r z_{t}
$$

where $r$ is the ratio of the bottom depth to the top depth.

Substituting equation 6 in 5 , with a simple rearrangement, we obtain the local-wavenumber equation for the contact model with finite depth extent as

$$
l w_{(h=0)}=\left(1+\frac{1}{r}\right) \frac{1}{z_{t}} .
$$

From equations 7 and 2, the relationship between the structural index and the ratio of the basal depth to the top depth of the contact model is given by

$$
\eta=\left(\frac{1}{r}\right) .
$$

Equation 8 indicates that the range of the structural index value for the magnetic layer is between 0 and 1 (Figure 2). If the ratio is relatively large (e.g., $r=z_{b} / z_{t} \approx 16$ ) then the structural index will be close to 0 , whereas if the ratio is relatively small (e.g., $r=z_{b} / z_{t} \approx 1$ ), the structural index will approach 1 .
Because an additional parameter $\left(z_{b}\right)$ has been introduced to the local-wavenumber equation, an estimate of one depth cannot be obtained without a prior estimation of the other. It should be noted that in this approach we only make use of the peak value of the local wavenumber to estimate the depth to source. Thus, for this simple model, equations 8 and 2 are accurate for any values of $z_{t}$ and $z_{b}$. This method does not suffer from the difficulties in using noninteger structural index values that have been reported for methods such as Euler deconvolution (Reid et al., 1990).

We have also assessed the magnitude of the depth error from using the infinite depth extent contact model. In Figure 3, we plot the true depth to the top of a magnetic contact versus the depth extent of the magnetic layer and we show, using color, the depth error generated using the infinite-depth model. For example, for a crustal contact beneath a sedimentary basin $2-\mathrm{km}$ deep (top to magnetic basement $=2 \mathrm{~km}$ ) in an area where the magnetic layer is $35-\mathrm{km}$ thick, the error

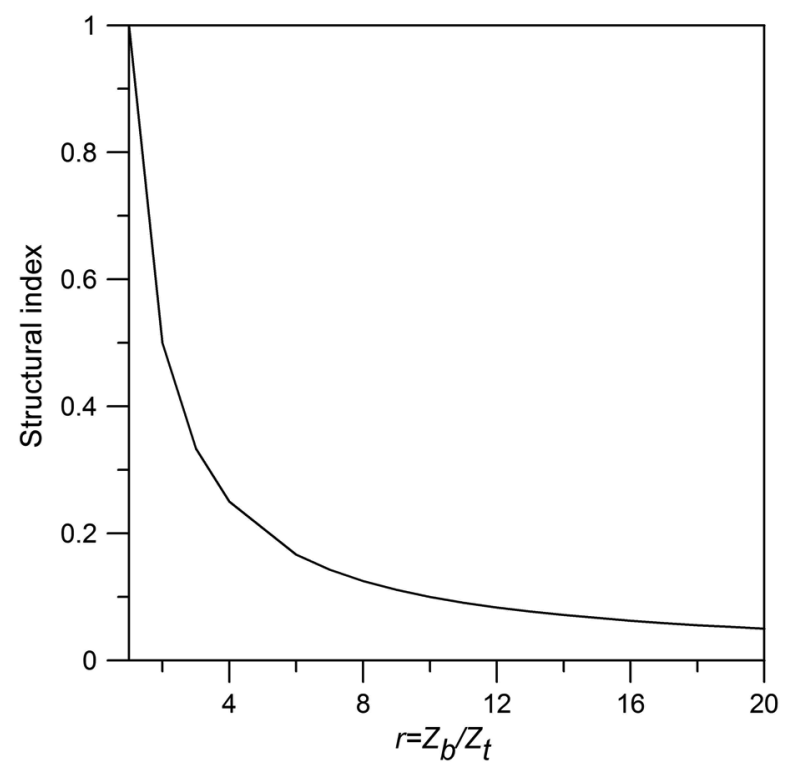

Figure 2. The relationship between the ratio of $Z_{b} / Z_{t}$ with the structural index for the vertical contact model.

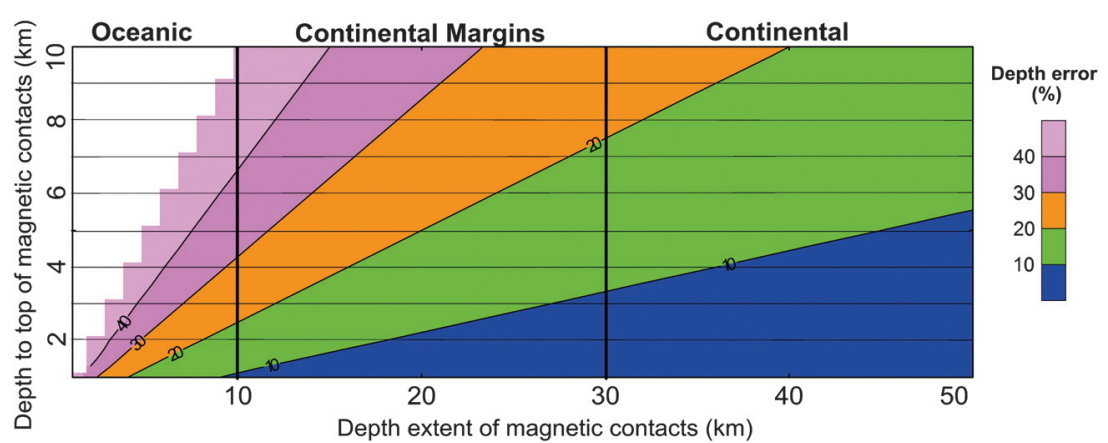

Figure 3. Error analysis for estimating the depth to the top of magnetic contacts while incorrectly assuming infinite depth extent. 
is in the blue area of Figure 3, which represents an underestimated depth error $<10 \%$. For a continental margin setting, however, where the top of the magnetic basement may be only $6-\mathrm{km}$ below the sea surface and the magnetic basement layer is 13-km thick, an infinitecontact model depth estimate will be $30 \%$ to $40 \%$ underestimated.

For the contact model, a ratio of $1: 10$ for $z_{t}: z_{b}$ gives a depth underestimation error of $\sim 9 \%$. Thus, a ratio of $\sim 1: 10$ could be considered as an indication of the situations in which the error will be significant. If one were equating the Curie depth with the magnetic bottom of the source, then Figure 3 shows that the validity of the assumption can be approximately met $(<10 \%$ error) in the continental regime and in continental margins where the depth to the top is small. As expected, the infinite depth extent assumption is not valid for most oceanic situations and results in large depth errors, especially in the normal ocean basin scenario in which the depth to the top of the magnetic layer (often close to the bathymetric depth) is large. The fact that the depths from the infinite-contact model are always underestimated suggests that to avoid systematic errors, it is generally best to make an estimate of the depth to the bottom of the magnetic layer.
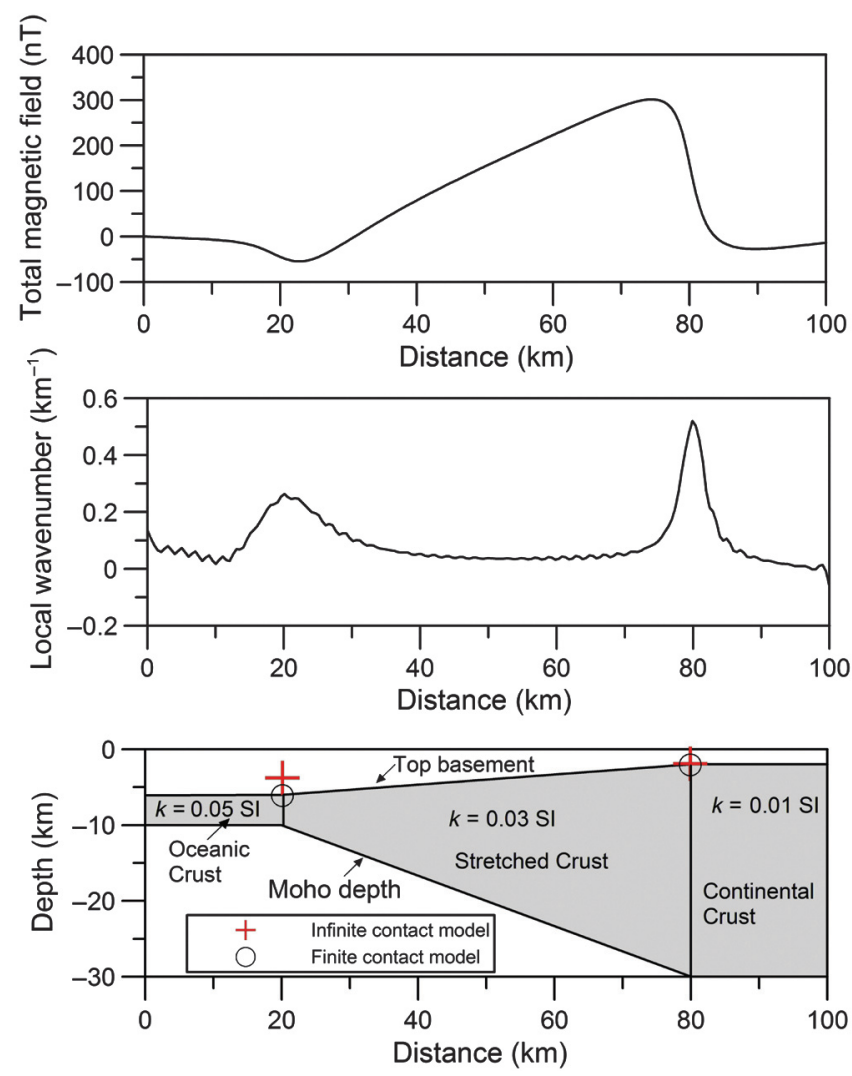

Figure 4. Continental margin model with vertical magnetic contacts. The cross symbols are the solutions using the infinite-contact model, and the circle symbols are the solutions using the finite-contact model.

\section{Continental margin model example}

To test these ideas, we initially applied the method to a synthetic continental margin model (lower panel of Figure 4), in which crustal magnetic susceptibility increases from 0.01 SI in the continental shield to $0.05 \mathrm{SI}$ in the oceanic crust. This simple crustal model assumes that the depth to the Curie $\left(580^{\circ} \mathrm{C}\right)$ isotherm extends to at least the Moho; thus, the entire crust is magnetic. This will not be the case in general, especially in regions of high heat flow where the Curie isotherm lies in the crust. If, on the other hand, the Curie isotherm extends into the upper mantle, then the Moho would remain the magnetic limit in the model because it is generally considered that the petrology of the upper mantle has an absence of magnetite or other common magnetic minerals with high Curie points and is thus nonmagnetic. The upper panel of Figure 4 shows the calculated magnetic anomaly, and the middle panel of Figure 4 shows the local wavenumber calculated using the fast Fourier transform method. First, we applied the conventional local-wavenumber method assuming an infinite-contact model (equation 2). Then, we applied the modified local-wavenumber method (equation 5) assuming that we have accurate information about the depth to the bottom. The estimates of the top basement depth using the conventional local-wavenumber method (red cross symbol) and the present approach (black open circle symbol) are very close over the continent where the crust is relatively thick and the depth to basement is shallow. However, when the depth to basement is 6-km deep at the stretched continent-ocean boundary, the depth estimate based on the infinitecontact model is underestimated by $2.2 \mathrm{~km}$ - a $36 \%$ error. For depths from the finite-contact model, on the other hand, the estimated depth to the top is close to the model depth.

\section{Example from the central Red Sea}

We demonstrate the practical utility of the localwavenumber finite-contact method using a magnetic profile (A-B) over the central Red Sea (Figure 5). The central and southern parts of the Red Sea are considered to be an embryonic ocean with oceanic crust along the axis, whereas the northern Red Sea is considered to be an active continental rift, in its late stage prior to the breakup and formation of oceanic crust. Rifting initiated around $30 \mathrm{Ma}$ (Bosworth and Burke, 2005) with movement of the Arabian plate northeastward away from the Nubian (African) plate to the southwest. In the central Red Sea, the crust is thin, especially along the axis where the crust is interpreted as oceanic with an age of $5 \mathrm{Ma}$ and younger (Cochran and Karner, 2007); hence, magnetic depth estimates with a finite depth extent would be expected to have a significantly detrimental impact on results. We used magnetic data from Getech's African magnetic compilation (Figure 5). The data are available as a grid with $1-\mathrm{km}$ cell size and are presented at a height of $1 \mathrm{~km}$ above land or seasurface-based on upward continuation as appropriate. 
Figure 6 shows the extracted magnetic anomaly and water depth for profile A-B. This profile was chosen to cross the area where the magnetic anomalies are most continuous in a ridge-parallel direction, such that the $2 \mathrm{D}$ approach used here is most valid. The highest amplitude anomalies are over the central oceanic part of the profile as expected. Before calculating the local wavenumber, the data were upward continued an additional $1 \mathrm{~km}$ (i.e., data $2 \mathrm{~km}$ above sea level) to reduce the effect of the noise and improve the stability of the depth estimates. Other enhancement filters such as low pass could be used. However, such filters can affect the accuracy of the results by providing deeper depths. Using the upward continuation filter has the advantage that the continuation distance is known and can be subtracted from the estimated depth. The local-wavenumber profile (upper panel of Figure 7) shows five definite symmetric zones (C1 to C5) characterized by different patterns representing different depth ranges and/or different crustal types. $\mathrm{C} 1$ and $\mathrm{C} 5$ are located in the coastal areas on continental crust of the African and Arabian plates. The width of the local-wavenumber anomalies in these zones is narrow with relatively high amplitude indicating shallow depth to basement expected on the rifted continental edge. The central zone C3 is associated with the rift axis and correlates very well with the area of deepest bathymetry (Figure 6). With the spreading rate of the central Red Sea segment $\left(\sim 1.3 \mathrm{~cm} / \mathrm{yr}\right.$ for about $19^{\circ}$ north; Chu and Gordon, 1998), the central feature of zone C3 corresponds with the Brunhes Chron or the present normal polarity epoch, which has lasted for the past 780,000 years (Cande and Kent, 1992), and the edges of the $\mathrm{C} 3$ zone correspond to $5 \mathrm{Ma}$ of spreading (Cochran and Karner, 2007). With the same spreading rate, the local-wavenumber highs in this central zone appear to correspond to distinct seafloor spreading anomalies. For example, the two peaks surrounding the central peak correspond to the magnetic polarity Chron 2A (1.78 Ma) and the two peaks on the ends of the central zone C3 appear to be related to Chron 3 (5 Ma). There are several reversals between these two epochs, and some of the longer ones appear to be indicated by the peaks of the local-wavenumber features in the central zone, C3. Zones $\mathrm{C} 2$ and $\mathrm{C} 4$ are characterized by broader, more variable, lower amplitude localwavenumber anomalies. They exhibit symmetry about the central anomaly, and the local-wavenumber peaks located at about 90 and $210 \mathrm{~km}$ could be related to Chron 5 (10 Ma). The use of the local-wavenumber technique for identifying seafloor spreading features is novel, and detailed analysis of

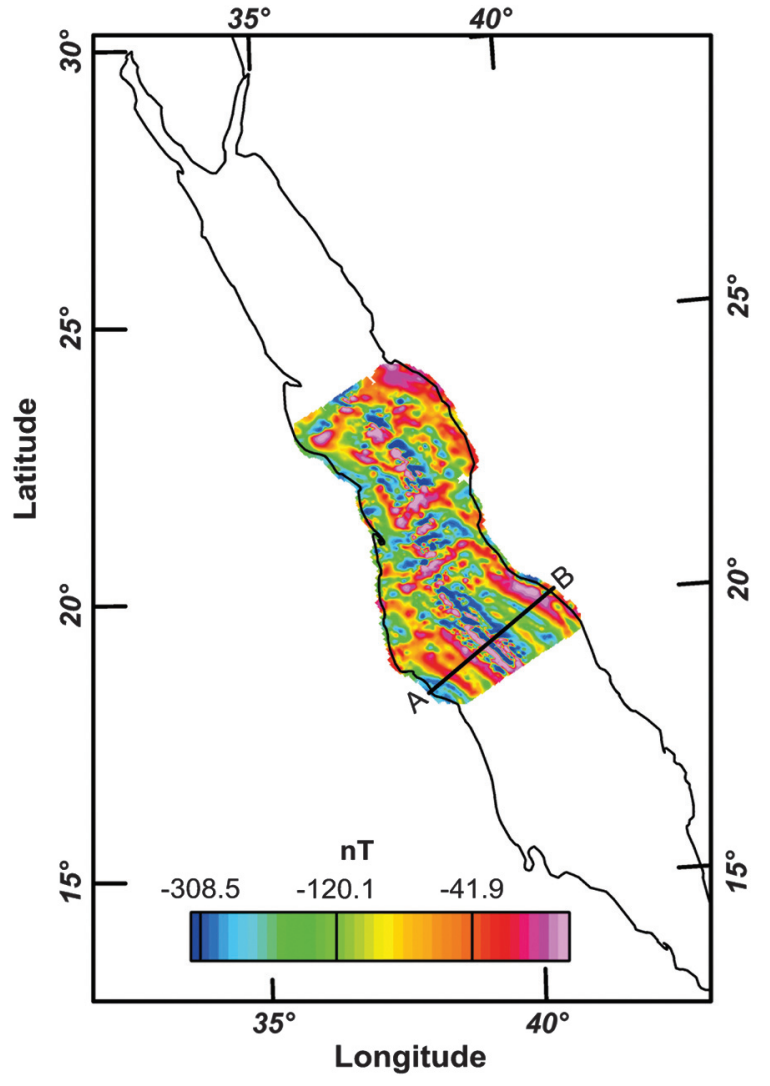

Figure 5. Location of a magnetic profile A-B cutting the central Red Sea rift from Sudan to Saudi Arabia.
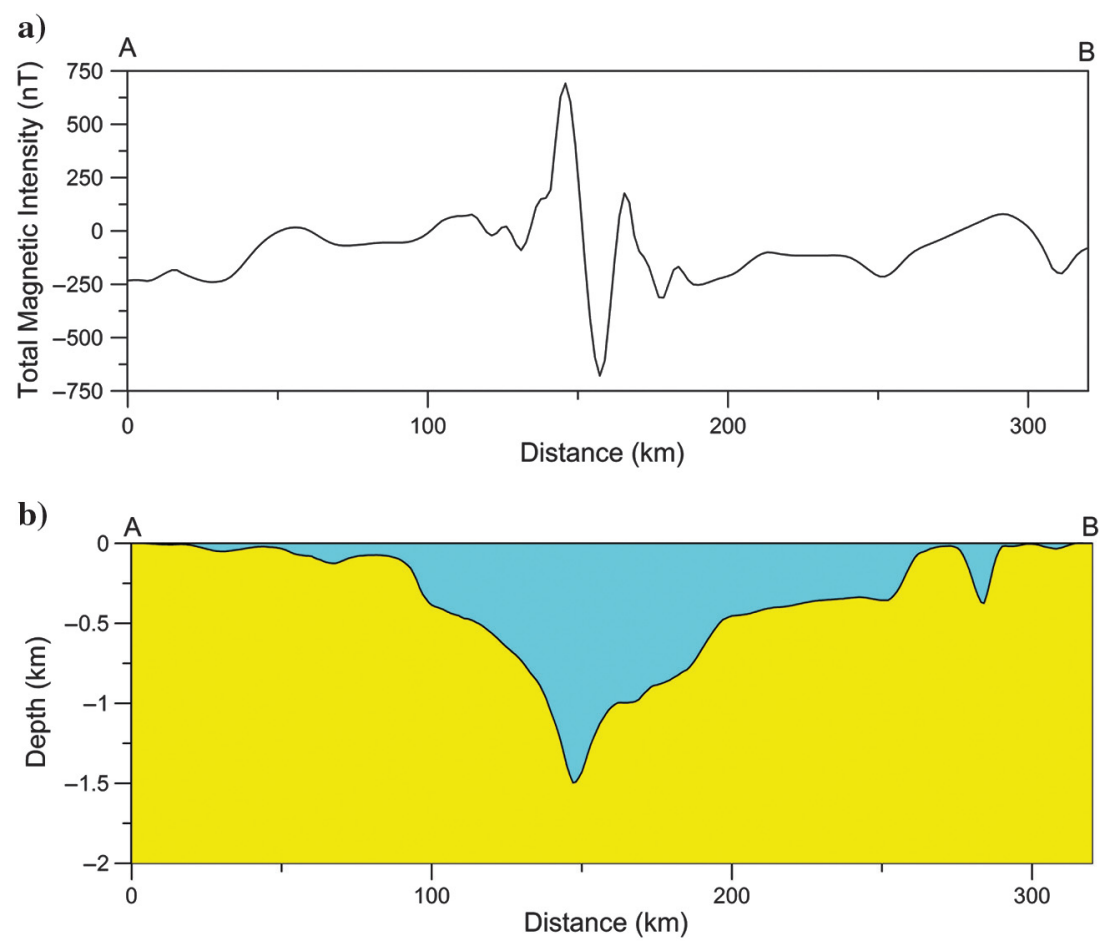

Figure 6. (a) Total magnetic intensity for a profile trending NE across the central Red Sea. (b) Public domain bathymetry along the profile A-B. Vertical exaggeration $50: 1$. 
magnetic sources could lead to fundamental knowledge about the nature, processes, and mechanisms of continental rifting. Thus, more work is underway to determine whether these features are associated with seafloor spreading stripes and anomalies or intermittent intrusive activity along with the seafloor spreading. Zones C2 and C4 are less symmetric about the center and are likely associated with intrusive activity in the stretched continental margin.

We estimated the depth using the conventional local wavenumber (equation 2) and the new finite local wavenumber (equation 5). We defined $z_{b}$ based on Moho depths determined from the 3D inversion of gravity anomaly data constrained by seismic results (Salem et al., 2013). Because the depth of the magnetic sources is controlled by the Curie temperature of magnetic minerals, we also computed the depth of the $580^{\circ} \mathrm{C}$ isotherm (the Curie temperature of magnetite). We used the half-space model (Turcotte and Schubert, 1982; Fowler, 2005), which is similar to the plate model for determining temperatures in the lithosphere younger than $50 \mathrm{Ma}$ (Stein and Stein, 1992). At $0 \mathrm{Ma}$, none of the thermal boundary layer models are correct for predicting temperatures in the crust where the central magnetic anomaly and magnetized zone are present; clearly, the rocks are magnetic and are at a temperature lower than their Curie temperature at least in oceanic layer 2 (Bleil and Petersen, 1983). The rest of the Curie isotherm corresponds to our determination of the Moho
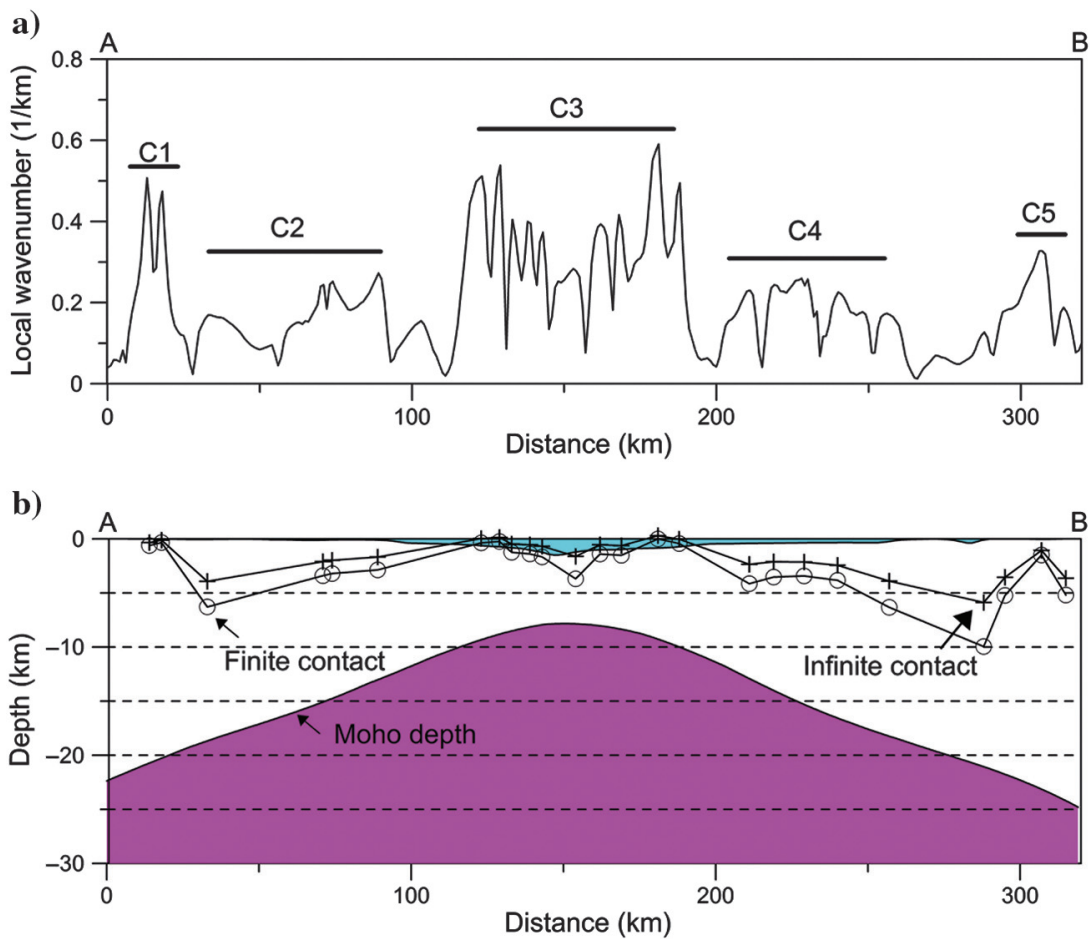

Figure 7. (a) The local wavenumber of the magnetic data of Figure 6a after upward continuing the data by a distance of $1 \mathrm{~km}$ (elevation of $2 \mathrm{~km}$ in total). (b) The depth-to-basement results assuming infinite-contact (cross symbol) and finite-contact (circle symbol) models and the depth to the bottom of the magnetic layer defined by the depth to Moho, from Salem et al. (2013). across the width of the Red Sea rift at this latitude. Thus, the Moho, and in this case equivalently the Curie isotherm of magnetite, is taken to define the bottom of the magnetic sources in our model.

We validated the estimate of the depth to source by comparison with bathymetry (Figure 6b) as a constraint on the shallowest solutions that may be expected. The results (Figure 7b) indicate that the depth solutions associated with the finite local-wavenumber approach are demonstrably better because several of the conventional results lie within the water column (i.e., underestimated depth).

To use these new results, we attempted to estimate values of effective susceptibility across the central Red Sea. This will help in understanding the nature of the crust in the area. We constructed a model with a magnetic layer involving numerous vertical-sided blocks (shown in Figure 8c). The top of the magnetic layer was obtained from our finite local-wavenumber solutions. The bottom of the magnetic layer was assumed to be equivalent to the Moho (Salem et al., 2013) or the Curie isotherm as discussed above. Additional contacts were added between the local-wavenumber peaks to allow better fitting of the magnetic data. These additional contacts may not appear as separate peaks in the local-wavenumber profile due to interference of adjacent anomalies. Next, we inverted the observed magnetic data using GM-SYS software $\left(\right.$ Geosoft $\left.^{\mathrm{TM}}\right)$ to estimate the effective susceptibility (the middle panel of Figure 8) for each block. For the past $30 \mathrm{Ma}$, the ambient field inclination and declination in the region are expected to be similar to the present. Remanent magnetization is not considered in this model, and so some of the downward excursions of the effective susceptibility are associated with reversely magnetized units; other lows may be related to intrusions of varying relative magnetization. The estimated effective susceptibility is very high in the rift area (up to $0.45 \mathrm{SI}$ ), in agreement with modeling results of Izzeldin (1987), who finds effective susceptibility varying between 0.01 and $0.06 \mathrm{cgs}$ units $(0.12$ and $0.75 \mathrm{SI})$. The strong magnetization of layer $2 \mathrm{~A}$ of the central zone and its rapid tenfold decrease in $20 \mathrm{Ma}$ due to low-temperature oxidation (Bleil and Petersen, 1983) are also apparent in the Red Sea rifting and spreading. However, the Red Sea rift is relatively young $(<5 \mathrm{Ma})$, and remanent magnetization will be nearly parallel or antiparallel to induced magnetization. Thus, the vector sum of induced and remanent magnetization can be considered equivalent to induced magnetization with a scalar "effective susceptibility." Across oceanic magnetic stripes, effective 


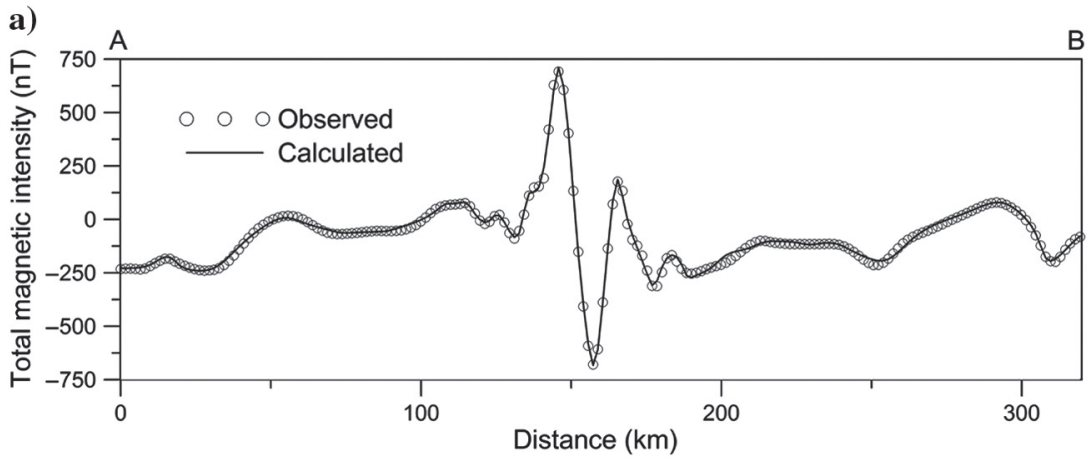

b)

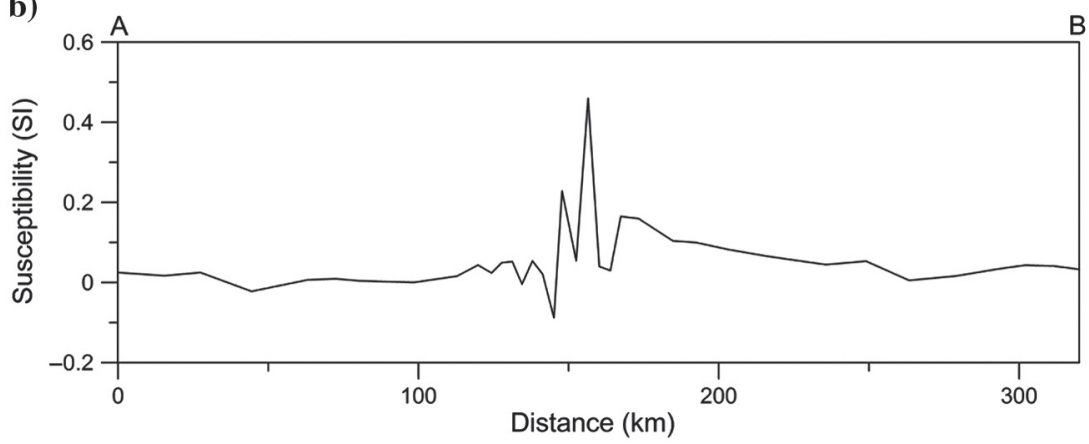

c)

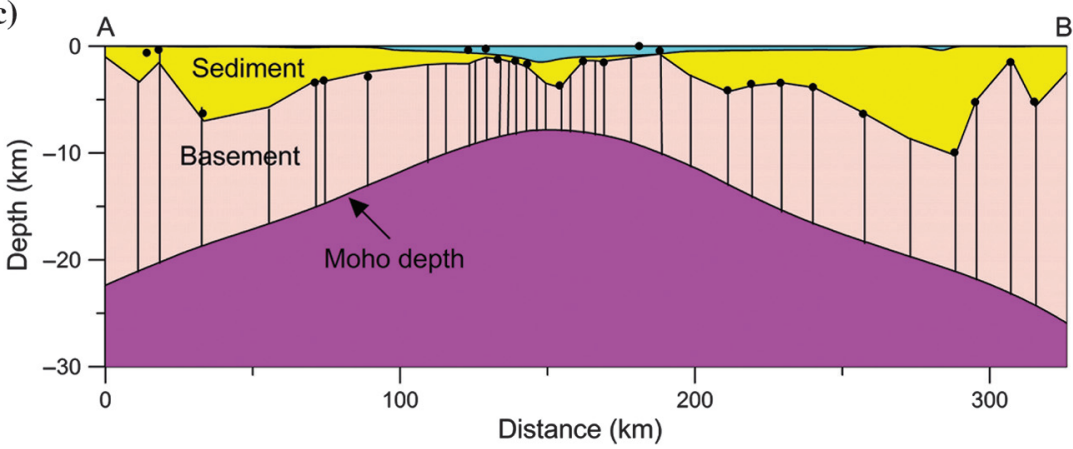

Figure 8. (a) The fit of the calculated magnetic anomaly and the observed anomaly. (b) The basement susceptibility associated with basement blocks. (c) The basement model with vertical contacts. Black circles are local-wavenumber solutions using the finite-contact model.

susceptibility may occasionally be negative. The average effective susceptibility value for the stretched crust on the African side is $0.02 \mathrm{SI}$ indicating that the crust is partially dominated by diabasic rocks. Similarly, on the eastern side of the rift, the average effective susceptibility is $0.05 \mathrm{SI}$, indicating the dominance of diabasic rocks.

\section{Conclusions}

This study demonstrates that the finite depth extent of a vertical contact has a significant impact on magnetic depth-to-source calculations using the local-wavenumber method. Assuming an infinite depth extent leads to significant errors in estimated depth in areas of thin crust and shallow Moho, or in areas of high heat flow, where the Curie isotherm is relatively shallow. The finite depth modification to the local-wavenumber approach always gives solutions that are deeper than the standard approach. The use of the finite-depth approach is required in the central Red Sea, where the crust is very thin and the Curie isotherm is elevated. In other areas where the Curie isotherm is not known, it is important to include at least the Moho in the localwavenumber calculation to limit the depth extent and improve estimates of depth to magnetic sources. Direct estimates of the Curie depth using magnetic and/or heat flow data can thus be valuable inputs to this process. We have also discovered in this study that the localwavenumber anomalies correlate well with major seafloor spreading magnetic reversal epochs in the central Red Sea segment.

\section{Acknowledgments}

We are grateful for comments by the associate editor, anonymous reviewers, and the editor Y. Sun, which helped to improve the paper. We are also indebted to S. Campbell for his discussion and comments on the manuscript. D. Ravat thanks the United States National Science Foundation (grant no. EAR-1246921) for his participation in this study.

\section{References}

Blakely, R. J., 1995, Potential theory in gravity and magnetic applications: Cambridge University Press.

Bleil, U., and N. Petersen, 1983, Variations in magnetization intensity and low-temperature titanomagnetite oxidation of ocean floor basalts: Nature, 301, 384388, doi: 10.1038/301384a0.

Bosworth, B., and K. Burke, 2005, Evolution of the Red Sea - Gulf of Aden Rift System: in P. Post, N. Rosen, D. Olson, S. L. Palmes, K. T. Lyons, and G. B. Newton, eds., 25th Annual GCSSEPM Foundation Bob F. Perkins Research Conference: Petroleum Systems of Divergent Continental Margin Basins, GCSSEPM, 342-372.

Cande, S. C., and D. V. Kent, 1992, A new geomagnetic polarity time scale for the Late Cretaceous and Cenozoic: Journal of Geophysical Research, 97, 13917-13951, doi: 10.1029/92JB01202.

Cochran, J. R., and G. D. Karner, 2007, Constraints on the deformation and rupturing of continental lithosphere of the Red Sea, in imaging, mapping and modeling continental lithosphere extension and breakup: Geological Society Publication, 282, 265-289. 
Chu, D., and R. G. Gordon, 1998, Current plate motions across the Red Sea: Geophysical Journal International, 135, 313-328, doi: 10.1046/j.1365-246X.1998.00658.x.

Flanagan, G., and J. E. Bain, 2013, Improvements in magnetic depth estimation: Application of depth and width extent nomographs to standard depth estimation techniques: First Break, 31, 41-51.

Fowler, C. M. R., 2005, The solid earth: Cambridge University Press.

Izzeldin, A. Y., 1987, Seismic, gravity and magnetic surveys in the central part of the Red Sea: Their interpretation and implications for the structure and evolution of the Red Sea: Tectonophysics, 143, 269-306, doi: 10.1016/ 0040-1951(87)90214-9.

Lee, M., B. Morris, and H. Uglade, 2010, Effect of signal amplitude on magnetic depth estimations: The Leading Edge, 29, 672-677, doi: 10.1190/1.3447778.

Nabighian, M. N., 1972, The analytic signal of twodimensional magnetic bodies with polygonal crosssection: Its properties and use for automated anomaly interpretation: Geophysics 37, 507-517, doi: 10.1190/1 .1440276.

Reid, A. B., J. M. Allsop, H. Granser, A. J. Millet, and I. W. Somerton, 1990, Magnetic interpretation in three dimensions using Euler deconvolution: Geophysics, 55, 80-91.

Salem, A., and R. S. Smith, 2005, Depth and structural index from the normalized local wavenumber of 2D magnetic anomalies: Geophysical Prospecting, 51, 83-89.

Salem, A., C. Green, S. Campbell, J. D. Fairhead, L. Cascone, and L. Moorhead, 2013, Moho depth and sediment thickness estimation beneath the Red Sea derived from satellite and terrestrial gravity data: Geophysics, 78, no. 5, G89-G101, doi: 10.1190/geo2012-0150.1.
Salem, A., S. Williams, J. D. Fairhead, D. Ravat, and R. Smith, 2007, Tilt-Depth method: A simple depth estimation method using first-order magnetic derivatives: The Leading Edge, 26, 1502-1505, doi: 10.1190/1.2821934.

Salem, A., S. Williams, E. Samson, J. D. Fairhead, D. Ravat, and R. J. Blakely, 2010, Sedimentary basins reconnaissance using the magnetic tilt-depth method: Exploration Geophysics, 41, 198-209, doi: 10.1071/ EG10007.

Stein, C. A., and S. Stein, 1992, A model for the global variation in oceanic depth and heat flow with lithospheric age: Nature, 359, 123-129, doi: 10.1038/359123a0.

Thompson, D. T., 1982, EULDPH: A new technique for making computer-assisted depth estimates from magnetic data: Geophysics, 47, 31-37.

Thurston, J. B., and R. S. Smith, 1997, Automatic conversion of magnetic data to depth, dip, and susceptibility contrast using the SPI method: Geophysics, 62, 807813, doi: 10.1190/1.1444190.

Turcotte, D. L., and G. Schubert, 1982, Geodynamics: Applications of continuum physics to geological problems: John Wiley \& Sons.

Wasilewski, P. J., and M. A. Mayhew, 1992, The Moho as a magnetic boundary revisited: Geophysical Research Letters, 19, 2259-2262.

Wasilewski, P. J., H. H. Thomas, and M. A. Mayhew, 1979, Moho as a magnetic boundary: Geophysical Research Letters, 6, 541-544.

Biographies and photographs of the authors are not available. 